IOS Press

\title{
The actual and potential impact of the novel 2019 coronavirus on pediatric rehabilitation: A commentary and review of its effects and potential disparate influence on Black, Latinx and Native American marginalized populations in the United States
}

\author{
Maurice G. Sholas \\ Principal and Pediatric Physiatrist, Sholas Medical Consulting, LLC, PO Box 8103, New Orleans, LA, 70182-8103, \\ USA \\ Tel.: +1 504322 2886; E-mail: SholasMedicalConsulting@gmail.com
}

\begin{abstract}
The COVID-19 pandemic has had a significant health impact around the world. In the United States, there has been a difference in infection and death rates for Black Americans and other marginalized groups as compared to White Americans. Although children do not seem to be suffering infection, morbidity and mortality to the same degree as adults, there is concern that COVID-19 could have a disparate impact on children with acquired or congenital disabilities when analyzed through the lens of race and equity. The possibility that there could be a differential effect on rehabilitation services relates to: the risk of familial/parental exposure leading to secondary infection, the negative economic impact of public health measures required to control disease spread, and the pre-existing social factors that impact access to healthcare. Finally, the psychosocial stresses imposed by COVID-19 inflame risk factors for non-accidental injury, which could lead to an increased need for pediatric rehabilitation services in vulnerable populations. It is critical that individual providers, as well as the health systems in which they practice, actively focus on mitigating personal and systemic causes of racial and ethnic health outcome disparities. These efforts need to move beyond a race neutral construct to specifically anti-racist activity.
\end{abstract}

Keywords: Health disparities, equity, coronavirus, COVID-19, pediatrics, marginalized population, disability, rehabilitation, function, Black children, Latino children, Native American children

\section{Introduction}

A novel coronavirus presented and began infecting humans, leading to a worldwide pandemic starting in 2019. The virus caused a severe respiratory syndrome in those most notably affected. As such, it was named severe acute respiratory syndrome coronavirus
2 (SARS-CoV-2) by the International Committee on Taxonomy of Viruses. SARS-CoV-2 is the virus that causes the coronavirus disease. Thus the disease is labeled COVID-19 for the virus and its year of origin. The global COVID-19 pandemic originated in the Wuhan province of China, but ultimately impacted the United States along with many developed and developing na- 
tions. As the data emerges, public health professionals sounded the alarm that the pandemic could have a disparate impact on vulnerable populations [1]. In the United States, early data reported from multiple jurisdictions showed that there was a disproportionate impact of this novel coronavirus when measuring death rates and infection rates in Black, Native American and Latinx persons when compared to White populations in multiple areas of the country [2]. These differences among vulnerable communities reflect known health outcome disparities in other care areas and disease processes.

Health disparities are also present at baseline in rehabilitation care. For example, there are notable racial and ethnic disparities in pain management resulting in Black and Latinx patients being less likely to get analgesia for acute pain than Whites [3]. Black and Latinx children requiring rehabilitation services following complex traumatic injuries were both less likely to be discharged to rehabilitation facilities and less likely to receive rehabilitation services after discharge from the acute care hospital [4]. The same was shown for pediatric Latinx populations for outpatient services after brain injury [5]. There is also compelling data that Native American and Native Alaskan children do not have the same degree of functional improvement from rehabilitation services as White children [6]. As such, there is a concern that these disparities, present in many disease states in addition to those requiring pediatric rehabilitation, will continue in COVID-19 related cases impacting functional outcomes and services. There is added concern that if the novel coronavirus takes hold in children with disabilities, this effect could be magnified in children from marginalized groups. This publication seeks to review the available data, elucidate areas of concern, and propose potential solutions to combat health disparities in the COVID-19 pandemic that affect the Pediatric Rehabilitation treatment diaspora.

\section{Risk of infection}

In the United States, like many countries, hospitals and healthcare systems modified their patient care plans in response to COVID-19. Outpatient visits deemed elective were postponed and non-essential surgical/procedural interventions were cancelled. State Medical Boards, in coordination with state government health departments, codified regulatory changes requiring a halt to elective and/or non-essential procedures. These actions were taken primarily to preserve per- sonal protective equipment, which was in short supply in key healthcare service areas. A secondary effect was to decrease the risk of frequent users of healthcare systems to coronavirus exposure and subsequent infection. Therefore, medically stable children with physical disabilities have been able to avoid leaving their homes for clinician visits. In addition, school closures across the country removed the other major reason children with disabilities leave the home. As such, patients with acquired or congenital disabilities are among the most thoroughly compliant with public health measures promoting sheltering at home and social distancing to control the spread of the virus.

The risk of children with disabilities contracting coronavirus infection is directly related to caregivers and/or family members that share their living arrangements. Household transmission data informs that risk. The phenomena of a non-infected person, living with a COVID-19 infected person, and becoming infected is called secondary transmission or the secondary attack rate. Data from the outbreak epicenter in China shows that this novel coronavirus has a household transmission rate that is higher than other coronaviruses like Middle-East Respiratory Syndrome and Severe Acute Respiratory Syndrome [7]. It is also higher than the common flu [7]. The relative risk varies depending on the relationship of the secondarily affected to the ill family member. The secondary attack rate for a spouse of an infected person is $27.8 \%$ whereas that for a child of the infected person is $4 \%$ [7]. In addition, the virulence of the virus in children overall is much reduced compared to adults [8]. This is reflected in the relatively low pediatric infection rate and exceptionally rare pediatric fatality as compared to adults. The social isolation inherent in the lives of children with physical disabilities [9] as well as their immaturity, potentially make these medically vulnerable children relatively protected against coronavirus infection. The risk of infection may be small, but it is not zero, and it is likely influenced by societal factors impacting the wellness of marginalized communities.

\section{Secondary impact}

The public heath response to control the spread of COVID-19 has centered on some key actions:

- Physical distancing/quarantine

- Travel restrictions

- Effective use of personal protective equipment

- Disease testing and tracing of infected individuals 


\section{- Healthcare capacity optimization}

These actions have potential financial and health impacts that affect children with disabilities. First, the ability to physically distance and shelter at home implies privilege. According to the US Bureau of Labor and Statistics, in 2017-2018 less than one in five black workers and nearly one in six Latinx workers were able to work from home [10]. That translates to nearly 8 in 10 workers from these marginalized communities who are not able to avoid community exposure as a means of protecting themselves and their children in the household from transmission. Higher-wage workers are six times more likely to be able to work from home as compared to lower-wage workers. This means the distribution of the children suffering from secondary attack of COVID infection will be inequitable. Race and socio-economic factors will leave differing populations of children with disabilities at various levels of risk based on the employment of their parent/guardian.

Second, COVID-19 mitigation strategies affect employment. According to the US Department of Labor, during the months of March and April 2020, there were 26.5 million initial jobless claims adding to the $7.1 \mathrm{mil}-$ lion Americans already unemployed. This figure of 33 million unemployed Americans is the highest level recorded since 1934. Families earning through manual labor and the service, restaurant, hospitality and tourism industries contribute to these data. Thus, children in those households may be forced from commercial insurance to public insurance. This would have a negative impact on access to pediatric rehab services. Insurance-based disparities cause children to be less likely to find adequate therapy and cause them to wait longer to receive services than those with commercial insurance [11]. The loss of livelihood caused by measures to control the spread of COVID-19 will change resources to support rehabilitation services for children. These effects will not be felt equally across all communities.

Access to healthcare services is a social determinant of health impacted by COVID-19. Minority children, when compared to White children, were more likely to report to the emergency room as their usual source of care [12]. This demonstrates a health access issue by highlighting lower rates of relationships with primary care providers. Although children with physical disabilities have a broader treatment team than typically developing children, they are not exempt from larger demonstrated issues of access to a medical home. In addition, children from communities of lower socioeconomic status use public transportation and those with disabilities rely heavily on ride-sharing programs. These modes of transport compel more exposure to the riders than private transportation; thus, these children are at greater risk of exposure to COVID-19. A combination of factors affecting access to care and transportation may lead to ominous racial and ethnic disparities in exposure and access to treatment in children with disabilities exposed to COVID-19.

With families under financial stress from employment challenges, psychosocial stress from being isolated from their community support structure, and overall anxiety about the ongoing effects of COVID-19 pandemic, there is a risk of increasing domestic violence and abuse. In an April 2020 virtual meeting of physicians specializing in Pediatric Rehabilitation Medicine, there were anecdotal reports of increased referrals for non-accidental trauma affecting children. These physicians were seeing patients through inpatient rehabilitation centers, outpatient clinics and day treatment programs at centers in all regions of the United States. The data documenting those suspicions have not yet been collected. However, according to the Center for Disease Control's violence prevention initiative, familial stress, unemployment, economic stress, and increased isolation are risk factors for domestic/intimate partner violence. The American Psychological Association raised similar alarms noting a 2019 study of populations following Hurricane Harvey in Texas with higher rates of domestic violence and child abuse [13]. Clinicians treating children with acquired disabilities must have a heightened awareness of this increasing risk and actively prepare to accommodate for an increase in brain injury and complex trauma following this pandemic.

Finally, as ventilator availability becomes the ratelimiting step to increasing capacity to care for those most critically ill from COVID-19, ethical issues present for children with physical disabilities that require permanent ventilation to live. Neuromuscular disease is the most common reason a child uses home ventilation [14]. These patients are commonly under the care of pediatric rehabilitation medicine in addition to multi-specialty teams of providers. The United States, like many countries, has experienced a shortage of ventilators during the COVID pandemic with only 62,000 fully functional hospital ventilators, 98,000 basic hospital ventilators and 8,900 ventilators in a strategic stockpile to serve an estimated 2.4-21 million Americans who will require hospitalization [15]. Thus, the question becomes would there be a circumstance or situation that would compel repurposing of ventilators being used for chronically ill or chronically declining patients 
for those acutely ill with COVID-19? Current sentiment does not make that likely as such a withdrawal of resources would not be at the request of the patient, not be deemed medically futile, nor ever considered in the absence of the pandemic [15]. However, this has made communities chronically dependent on ventilators outside of the hospital setting worry that they could be deemed expendable. Those concerns of societal value are magnified for marginalized populations.

\section{Recommendations}

There is considerable concern that health inequity seen in many disease processes and interventions central to pediatric rehabilitation medicine may be exacerbated directly or indirectly by the COVID-19 pandemic. As a community of care providers, we must actively work against that. Causal factors for inequitable health outcomes are at both the "micro" (individual) and the "macro" (systemic) level. They include factors unique to the patient as well as ones that exist outside of them. As healthcare providers and leaders, we must spearhead active interventions at the personal, systemic and policy levels.

Each provider caring for diverse patients has to do the work of understanding and acknowledging unconscious bias. Validated measures like the Implicit Association Test are able to quantify the level of anti-black bias in an individual [16].

"Although overt discriminatory behavior in the United States may have declined in recent decades, covert discrimination and institutional bias are sustained by subtle, implicit attitudes that may influence provider behavior and treatment choices. As a result, patients of color may be kept waiting longer for assessment or treatment than their White counterparts, or providers may spend more time with White patients than with patients of color. In addition, providers may vary in the extent to which they collaborate with patients in systematic though nondeliberate ways, in considering treatment options based on patients' characteristics." [17]

There is criticism of this framing as it removes the onus on the individual for perpetuating harm by labeling it unintentional. This sentiment values the need to soothe the emotional response of the provider contributing to disparities when confronted with that reality over the negative health effects that internalized bias inflicts on marginalized patients. However, a problem unknown and unrecognized cannot be solved. So this personal responsibility to be actively anti-racist, not just race indifferent or neutral, starts with framing the problem on an individual level. There is a causal relationship between biased physician behavior and healthcare disparities [18]. It is the responsibility of every clinician to address that proactively and substantively. The COVID-19 response in Rehabilitation Medicine provides a chance to do so.

Systemic changes to address healthcare outcomes are more challenging to address. They are based in the Social Determinants of Health [19] and cover issues from community location and resources to family unit stability. The neighborhood and environment where patients live determine safety and ease of access to clinical treatment options. Mindfully planning the locations of future rehabilitation centers in communities following the pandemic can positively impact outcomes in marginalized groups. Patients are more likely to utilize and benefit from services they can obtain easily.

Family units require a modicum of health and capacity to provide for children with special healthcare needs. People of color receive a lesser quality of care in the US Health System [19]. In the time of COVID-19 there is a decrease in the baseline capacity for marginalized group members to accommodate the needs of children with disabilities. The powerful impact of COVID19 actively decreasing the overall health of families is compounded in communities of color. Beyond the family unit, communities are straining the resources used to support those in need. The family unit and social community is a critical determinant of health. Programs that promote healthy communities support the ability for them to foster wellness amongst its members. In an era of social/physical distancing the rituals and mores that allow communities to bond and celebrate togetherness, and mourn those lost, are removed. Pediatric rehabilitation patients have a drastically changed context of community support and belonging through no fault of their own. Empowered communities with access to quality information make better choices. Advocacy for marginalized groups to get needed resources must happen intentionally and in a way that "... disregard[s] the underlying effects of racial bias and racism" [20].

Education impacts health, and COVID-19 has shuttered schools all over the United States for an extended period of time. There is a future cost to the education of all children, especially those that need specialty equipment and/or specifically qualified educators to optimize learning. Plus, societal resistance to COVID-19 containment measures underscores how the science and 
mathematical concepts informing public health recommendations are not translating to large portions of the American public. Marginalized populations, with historic mistrust of traditional institutions of healthcare, are particularly vulnerable to factually and scientifically disingenuous health myths. It is the responsibility of all in the system to provide quality and accurate insight to our patients in a manner they can receive and incorporate.

Finally, economic stability is a social determinant of health and has been a recurring theme of this submission. COVID-19 has caused a skyrocketing unemployment rate and halted entire industries. Policies that provide living wages and financial resources are critical during the pandemic for communities to recover. They are especially important to children with disabilities from marginalized groups. Healthcare professionals have to embrace their role as influential citizens outside of just health related issues. Solutions mitigating the disparate effect of COVID-19 on pediatric rehabilitation must acknowledge and incorporate the patient's environment, overall family health, community context, education, and economic stability.

The work to decrease potential health disparities in pediatric rehabilitation requires active policy changes. Some have proposed to increase accountability by including health equity issues in ethics and professionalism requirements [21]. They propose incorporation of more humanities training at all levels of education, from the pre-medical years through post graduate training and in medical practice, in addition to enhancing ethical reflection and critical thinking skills [21]. There are challenges in adding policies acknowledged in the literature. A panel of research and clinical professionals connected in 2013 as the Pediatric Health Equity Collaborative. They clearly state that effective approaches to systematic factors precipitating bias will not be singular nor applied without modification to meet the needs of a given health system [22]. However, they recommend collecting several data points on the child and family unit and incorporating this information into the patient's electronic health record. These statistics will allow the tracking of reliable data for intentional quality improvement. A caution in planning policy interventions is to avoid focusing exclusively on individual-level challenges disabled children from marginalized groups face without giving attention to systemic-level problems that institutions can mitigate. The literature features articles that review interventions targeting individual-level factors to optimize healthiness and health behaviors, but there are not evaluations of the impact of system-level factors like increasing the diversity of the medical providers, planning locations of services such that they are easily accessible from the poorest zip codes by public transportation, and/or removing payer mix limitations that ration the number of public insurance patients allowed in a clinical service area. Members of Congress and organized medicine must use the lens of critical race theory to view the response to COVID-19 [20] and we in pediatric rehabilitation must blunt the potential impact on children from marginalized communities.

\section{Conclusion}

Patients cared for in the pediatric rehabilitation service diaspora have racial and ethnic outcome disparities. These disparities may be highlighted by the personal, social and financial impact of the COVID-19 pandemic. Although children with physical and functional disabilities may be relatively shielded from direct infection by their relative social isolation and pediatric status, they are vulnerable to poor outcomes with any infection that does take hold. Additionally, they are sensitive to systemic factors, like the social determinants of health, which could intensify disparities in healthiness and functional outcomes. To mitigate these elements, individual providers, health systems and policy interventions must be accountable and active. The goal is to ensure that marginalized communities, like Black, Latinx, and Native American children, do not continue to be disproportionately impacted in a negative way by this health catastrophe.

\section{Conflict of interest}

No conflicts of interest to disclose.

\section{References}

[1] Smith JA, Judd J. COVID-19: Vulnerability and the power of privilege in a pandemic. Health Promot J Austr. 2020 Apr; 31(2): 158-160. doi: 10.1002/hpja.333.

[2] Johns Hopkins University \& Medicine Coronavirus Resource Center. Baltimore [cited 2020 Apr 23]. Available from: www. coronavirus.jhu.edu.

[3] Lee P, Le Saux M, Siegel R, et al. Racial and ethnic disparities in the management of acute pain in US emergency departments: Meta-analysis and systematic review. Am J Emer Med. 2019 Sep; 37(9): 1770-1777. doi: 10.1016/j.ajem.2019.06.014.

[4] Shah AA, Zuberi M, Cornwell E, et al. Gaps in access to comprehensive rehabilitation following traumatic injuries in children: A nationwide examination. J Pediatr Surg. 2019 Nov; 54(11): 2369-2374. doi: 10.1016/j.jpedsurg.2019.06.001. 
[5] Moore M, Jimenez N, Rowhani-Rahbar A, et al. Availability of outpatient rehabilitation services and barriers to care for vulnerable populations after pediatric traumatic brain injury. Am J Phys Med Rehabil. 2016 Mar; 95(3): 204-213. doi: 10.1097/PHM.0000000000000362.

[6] Fuentes MM, Bjornson K, Christensen A, Harmon R, Apkon SD. Disparities in Functional Outcomes During Inpatient Rehabilitation Between American Indian/Alaska Native And White Children. J Health Care Poor Underserved. 2016; 27(3): 1080-96. doi: 10.1353/hpu.2016.0143.

[7] Li W, Zhang B, Lu J, et al. The characteristics of household transmission of COVID-19. Clin Infect Dis. 2020 Apr 17; ciaa450. doi: 10.1093/cid/ciaa450.

[8] Cristiani L, Mancino E, Matera L, et al. Will children revea their secret? The coronavirus dilemma. Eur Respir J. $2020 \mathrm{Apr}$ 23; 55(4): 2000749. doi: 10.1183/13993003.00749-2020.

[9] Tonkin BL, Ogilvie BD, Greenwood SA, Law MC, Anaby DR. The participation of children and youth with disabilities in activities outside of school: A scoping review. Can J Occup Ther 2014 Oct; 81(4): 226-236. doi: 10.1177/0008417414550998.

[10] Gould E, Shierholz H. Not Everybody Can Work From Home. Working Economics Blog. Economic Policy Institute. [cited 2020 Apr 23]. Available from: www.epi.org/blog.

[11] Fuentes MM, Thompson L, Quistberg DA, et al. Auditing access to outpatient rehabilitation services for children with traumatic brain injury and public insurance in washington state. Arch Phys Med Rehabil. 2017 Sep; 98(9): 1763-1770.e7. doi: 10.1016/j.apmr.2016.12.013.

[12] Shone LP, Dick AW, Brach C, et al. The role of race and ethnicity in the state children's health insurance program (SCHIP) in four states: Are there baseline disparities, and what do they mean for SCHIP? Pediatrics. 2003 Dec; 112 (6 Pt 2): e521.

[13] Abramson A. How COVID-19 May Increase Domestic Violence and Child Abuse. American Psychological Association. [cited 2020 Apr 23]. Available from: www.apa.org/topics/ covid-19/domestic-violence-child-abuse.
[14] Amirnovin R, Aghamohammadi S, Riley C, Woo MS, Del Castillo S. Analysis of a pediatric home mechanical ventilator population. Respir Care. 2018 May; 63(5): 558-564. doi: 10.4187/respcare.05644

[15] Truog RD, Mitchell C, Daley GQ. The toughest triage - allocating ventilators in a pandemic. N Engl J Med. 2020 May 21; 382(21): 1973-1975. doi: 10.1056/NEJMp2005689.

[16] Nosek BA, Smyth FL, Hansen JJ, et al. Pervasiveness and correlates of implicit attitudes and stereotypes. Eur Rev Soc Psychol. 2007; 18: 36-88

[17] Hall WJ, Chapman MV, Lee KM, et al. Implicit racial/ethnic bias among health professionals and its influence on health care outcomes: A systematic review. Am J Public Health. 2015 Dec; 105(12): e60-76. doi: 10.2105/AJPH.2015.302903.

[18] FitzGerald C, Hurst S. Implicit Bias in Healthcare Professionals: A Systematic Review. 2017 Mar 1; 18(1): 19. doi: 10.1186/s12910-017-0179-8.

[19] Braveman P, Egerter S, Williams DR. The social determinants of health: Coming of age. Annu Rev Public Health. 2011; 32 381-98. doi: 10.1146/annurev-publhealth-031210-101218.

[20] Chomilo N, Heard-Garris N, DeSliva M, Blackstock U. The Harm of a Colorblind Allocation of Scarce Resources. Health Affairs Blog. 2020. [cited 2020 Apr 30]. Available from: https://www.healthaffairs.org/do/10.1377/hblog20200428.904 804/full/.

[21] Doukas DJ, Kirch DG, Brigham TP, et al. Transforming educational accountability in medical ethics and humanities education towards professionalism. Acad Med. 2015 Jun; 90(6): 738-43. doi: 10.1097/ACM.0000000000000616.

[22] Tan-McGrory A, Bennett-AbuAyyash C, Gee S, et al. A Patient and Family Data Domain Collection Framework for Identifying Disparities in Pediatrics: Results from the Pediatric Health Equity Collaborative. 2018 Jan 31; 18(1): 18. doi: 10.1186/s12887-018-0993-2. 